\title{
COVID-19: An Aerosol's Point of View from Expiration to Transmission to Viral-mechanism
}

\author{
Ta-Chih Hsiaoo ${ }^{1 \#}$, Hsiao-Chi Chuang ${ }^{2,3,4 \#}$, Stephen M. Griffith ${ }^{5}$, Shui-Jen Chen ${ }^{6}$, Li-Hao Young ${ }^{7 *}$ \\ ${ }^{1}$ Graduate Institute of Environmental Engineering, National Taiwan University, Taipei 106032, Taiwan \\ ${ }^{2}$ School of Respiratory Therapy, College of Medicine, Taipei Medical University, Taipei 11031, Taiwan \\ ${ }^{3}$ Cell Physiology and Molecular Image Research Center, Wan Fang Hospital, Taipei Medical University, Taipei 11696, \\ Taiwan \\ ${ }^{4}$ Division of Pulmonary Medicine, Department of Internal Medicine, Shuang Ho Hospital, Taipei Medical University, \\ New Taipei City 23561, Taiwan \\ ${ }^{5}$ Department of Atmospheric Sciences, National Central University, Taoyuan 32001, Taiwan \\ ${ }^{6}$ Department of Environmental Science and Engineering, National Pingtung University of Science and Technology, \\ Pingtung 91201, Taiwan \\ ${ }^{7}$ Department of Occupational Safety and Health, College of Public Health, China Medical University, Taichung 40402, \\ Taiwan
}

\section{INTRODUCTION}

Coronavirus disease 2019 (COVID-19) emerged in Wuhan, China in late 2019, and soon unfolded as a global outbreak accompanied by declarations of a public health emergency of international concern (PHEIC) and later a pandemic from the World Health Organization (WHO). COVID-19 has resulted in 2,626,321 confirmed cases and 181,938 reported deaths worldwide (as of 14 April 2020). The underlying virus, severe acute respiratory syndrome coronavirus 2 (SARS-CoV-2), is a highly contagious novel coronavirus that transmits as an aerosol and threatens people of all ages, from infant to geriatric, while those with cardiovascular disease are recognized as particularly susceptible to more severe symptoms from SARSCoV-2 infection. WHO recommends using a mask to limit the COVID-19 outbreak; however, SARS-CoV-2 transmission models and its removal efficiency by wearing a mask remain unclear. Furthermore, differences in the field-specific definitions/terminologies related to transmission and mask usage tend to cause confusion and misunderstanding among both experts and the public.

\section{SARS-COV-2 AND ANGIOTENSIN-CONVERTING ENZYME 2}

SARS-CoV-2 is a positive-sense single-stranded RNA virus (Satija and Lal, 2007), with virion sizes ranging between 60 and $140 \mathrm{~nm}$ (Zou et al., 2020). These virions have been observed in a wide range of intracellular organelles (i.e., vesicles) after infection (Kim et al., 2020). For respiratory infection to occur, a sufficient amount of viable SARS-CoV-2 virions have to be contained in the "carrier" aerosols. The respiratory tract is the primary target for virus infection by droplet-aerosol transmission. In terms of functionality, the respiratory system is divided into conducting and respiratory zones, where the latter contains the alveoli and is thought to contain the cells most vulnerable to SARS-CoV-2 attack. Angiotensin-converting enzyme 2 (ACE2) on the cell membrane (e.g., of pneumocytes) provides the eventual entryway for SARS-CoV-2 (Hoffmann et al., 2020). The S1 domain of a coronavirus contains the ACE2 receptor-binding structure that is responsible for host cell entry (Li et al., 2005), while the S2 domain facilitates fusion between the cell and virus membrane that is required for cellular infiltration (Coutard et al., 2020). The spike glycoprotein (i.e., S1 domain) of SARS-CoV-2 has a higher affinity than other viruses for ACE2 (Vankadari and Wilce, 2020; Wrapp et al., 2020); therefore, ACE2 receptors provide a particularly sensitive adhesion site for SARS-CoV-2 to invade cells (Brake et al., 2020). Notably, in addition to the vulnerability of ACE2-bearing cells in the respiratory zone, a recent report indicated that ACE2 is prominently expressed in a transient secretory cell type in the subsegmental bronchial branches of lungs (Lukassen et al., 2020). If cells in the conducting zone

\footnotetext{
${ }^{*}$ Contributed equally as the first author.

${ }^{*}$ Corresponding author.

E-mail address: lhy@mail.cmu.edu.tw
} 
are also vulnerable to SARS-CoV-2 attack, then carrier aerosol sizes ranging between 0.5 and $2 \mu \mathrm{m}$ should be a concern. In other words, the SARS-CoV-2 "carrier" aerosol size is one of the important factors to consider in its path to pulmonary infection and thus for effective SARS-CoV-2 infection control.

\section{MODES OF TRANSMISSION: DROPLET VS. AIRBORNE}

According to WHO (2020b), SARS-CoV-2 is primarily transmitted by respiratory droplets produced by sneezing and coughing from an infected person, either directly when the droplets reach a person's nose, mouth or eyes, or indirectly when a person touches a droplet-contaminated surface or inanimate object (i.e., fomite) and then touches his/her nose, mouth or eyes. Thus, widely advocated preventive measures against SARS-CoV-2 infection, such as wearing masks, frequent hand washing and social distancing, are meant to minimize transmission from nearby infected persons. More recently, aerosol and hence airborne transmission was indicated as a plausible pathway to spread SARS-CoV-2 (van Doremalen et al., 2020), and has been inciting public concern, but also fostering insightful research endeavors. So, what is airborne transmission, and how is it different from droplet transmission?

In the field of infection control, airborne transmission specifically refers to the spread of infectious agents via droplet nuclei or smaller aerosols suspended in air (CDC, 2012), implicitly excluding the relatively larger droplets and seemingly at odds with the WHO statement. However, the transmission medium for both "droplets" and "droplet nuclei" is air, thus both are technically airborne carriers. Furthermore, in the field of aerosol science, droplets and droplet nuclei are both considered "aerosols" (i.e., tiny airborne solids or liquids ranging from a few nanometers to about $100 \mu \mathrm{m}$ in diameter). The major physical differences between droplets and droplet nuclei are their size and mixing state; droplets are relatively large and wet, whereas droplet nuclei are the by-product of larger droplets upon evaporation (i.e., drying) while suspended in air and thus are relatively small and dry. Aerosol-related studies on respiratory droplets and droplet nuclei were pioneered by Wells (1934), and have been followed by numerous other studies since then (e.g., Li et al., 2007; Eames et al., 2009; Vejerano and Marr, 2018).

To go further in-depth, respiratory droplets are saliva and secretions expelled (i.e., atomization) from the upper airway and through the mouth or nose during human expiratory activities such as coughing, sneezing, laughing, talking and even normal breathing (Yang et al., 2007; Morawska et al., 2009). Morawska et al. (2009) showed that the majority of droplets, by number, expelled from various breathing and mild throat clearing cough scenarios are mainly $<1 \mu \mathrm{m}$ in diameter. On the other hand, droplets from sneezing are considerably larger (Han et al., 2013). Respiratory droplets of concern in infection control are usually those larger than 5 or even $10 \mu \mathrm{m}$ in diameter, although vary with many human and environmental factors (Liu et al., 2017). Due to gravity, these relatively large droplets settle out to the floor or other surfaces within a few seconds, but the distance to which they travel depends on the specific expiratory activity and the surrounding airflow field. In revisiting the Wells evaporation-falling curve, Xie et al. (2007) showed that large droplets from sneezing could travel more than $6 \mathrm{~m}$, or $2 \mathrm{~m}$ from coughing and $1 \mathrm{~m}$ from breathing. Once expelled, respiratory droplets rapidly evaporate $(<1 \mathrm{~s})$ to an equilibrium size that is dependent on ambient relative humidity and original droplet size before falling away (Nicas et al., 2005; Xie et al., 2007; Morawska et al., 2009). As mentioned, respiratory droplets can even dry-out and transform into droplet nuclei, where the success of this transformation depends on the competing evaporation and gravitational settling rates.

Droplet nuclei are usually less than $5 \mu \mathrm{m}$ in diameter, and therefore can remain suspended in air for a longer period of time, and travel a further distance away. It is this transport characteristic that more accurately distinguishes airborne (droplet nuclei) transmission from droplet transmission. The spatial scale of outdoor airborne transmission can extend to a few kilometers, depending on the wind condition (Yu et al., 2004). Another unique characteristic associated with smaller droplet nuclei is the increased ease of inhalation deeper into the human respiratory tract, with decreasing particle size. Therefore, airborne transmission is of particular concern; however, the biological implications of airborne transmission in COVID-19 are currently still unclear.

With the above droplet and droplet nuclei characterizations laid out, how do we relate them to the current COVID-19 pandemic? First and foremost, it is critical to note that transmission is not the only factor determining whether or not a person will be infected by SARS-CoV-2. A comprehensive infection risk assessment demands more factors for consideration, including the virus (e.g., viability and infectious dose), infected person (e.g., viral load), transmission mode (droplet vs. airborne), aerosol processes (e.g., phase transformation and respiratory deposition), the medium (e.g., building ventilation and transport processes) and host (e.g., immune system and use of personal protection equipment), and integrates the risks from source to receptor. Consider a simplified example where larger wet droplets may carry more, viable viruses than relatively smaller dry droplet nuclei. The site and efficiency of respiratory deposition would be different for different droplet sizes; larger droplets would deposit more efficiently in the upper respiratory tract. The dose, and hence risk of infection, would then be related to the droplet or nuclei number concentration, the contained number of viable viruses, the deposition efficiency and the susceptibility of the deposited sites. With the risks tallied up, risk prioritization then considers the optimal allocation of limited resources and efforts to areas with the highest risk. If droplet transmission is truly the major pathway of SARS-CoV-2 transmission as stated by WHO, the focus of prevention and control should be given there accordingly, but the possibility of airborne transmission should be clarified and taken seriously as a transmission route in the meantime. Drawing a nominal dichotomy between respiratory droplets and droplet nuclei based on their physical size and mixing state, or judging the risk of infection based solely on transmission is an oversimplification. 


\section{MASKING THE PROBLEM OF COLLECTION EFFICIENCY}

At the outset of the COVID-19 outbreak, a central focus in some countries was already on the availability of masks, in particular N95 respirators ( $\geq 95 \%$ aerosol collection efficiency at $0.3 \mu \mathrm{m}$ ) (Lee et al., 2005) and surgical masks. The focus on masks was further highlighted with indication that SARS-CoV-2 virus could survive in the form of aerosols, generated by a Collison nebulizer and fed into a Goldberg drum, with a half-life of more than 1 hour (van Doremalen et al., 2020), and mask availability eventually became a worldwide concern as the COVID-19 outbreak spread to nearly every corner of the globe. While tight-fitting N95 respirators provide better protection against inhalation of airborne submicron- to nano-particles (Zhou and Cheng, 2016; Ollier, 2018; Lin et al., 2020), they are less comfortable to wear and are generally recommended to be reserved for medical professionals/health care workers during a pandemic (WHO, 2020a). Surgical masks, which are designed for avoiding direct exposure to large expiratory droplets, have been generally the most coveted protective wear during the COVID-19 outbreak, largely for having the opposite characteristics to the N95 respirator: they fit more loosely to the face, thus are more comfortable, and are low cost (7-10× per unit less than the N95 respirator). However, the collection efficiency of surgical masks is low and highly variable, ranging from $25-75 \%$ (Chen and Willeke, 1992), depending on the quality of the filter media, filtration velocity and particle size (Jung et al., 2014). Oberg and Brosseau (2008) further concluded surgical masks do not offer protection comparable with that of respiratory protective devices, since they seldom meet facial fit performance criteria. Thus, the surgical mask is often considered as a protection for others instead of for oneself. Nonetheless, worldwide trends have shown that countries with a higher percent of the population wearing masks (i.e., surgical masks) have less uncontrolled outbreaks of COVID-19. Thus, it begs the question: How do we square these seemingly incongruent findings of poor collection efficiency by surgical masks and their success rate at preventing the virus from spreading?

We believe the answer to that question is in part due to the reduction in expired air velocity, an often overlooked factor in the efficacy assessment of masks. Upon exhalation, the average expired air velocity may range from around $3.9 \mathrm{~m} \mathrm{~s}^{-1}$ to $11.7 \mathrm{~m} \mathrm{~s}^{-1}$ depending on whether the person is speaking, coughing, etc. (Chao et al., 2009). Increased expired air velocities correspond to an increased distance of microdroplet ejection away from our mouths. Recently, Bourouiba (2020) demonstrated that exhalations, sneezes, and coughs not only consist of large mucosalivary droplets with short-range trajectories, but are primarily made of cloud entraining droplets of various sizes. The local warm and moist condition of these expiration clouds can preserve the droplets, and with the assistance of the cloud's expiration momentum, promote their transport up to 7-8 m. Even under normal speech, Asadi et al. (2019) found that a non-negligible amount of particles with a geometric mean diameter of $1 \mu \mathrm{m}$ can be generated and emitted by a "fluid-film burst" mechanism in the larynx, where an abundant SARS-CoV-2 population may reside (Zou et al., 2020). By wearing a mask, we introduce a resistance barrier to these droplets, whose population on the other side of the mask barrier should inversely scale to the mask's collection efficiency. But what's more, the mask is also reducing the wearer's expired air velocity after the mask and thus having an additional containment effect by shrinking the effective ejection radius of the droplets that penetrate the mask filtration layers. In particular, coughing or sneezing increases the expired air velocity, which increases the impaction efficiency of the large droplets, but reduces the collection efficiency of the fine particles. While those particles are not contained by the mask, their risk of contributing to the airborne spread of the virus is severely reduced. Furthermore, the inhalation velocity on the outside of the mask is also attenuated, meaning the effective radius from which the mask wearer is drawing air is also dramatically reduced. In this letter, we are suggesting that collection efficiency should not be the only defining characteristic of efficacy when discussing the utility of different masks to prevent the spread of the virus. Rather the reduction in expired air velocity should be viewed as a common denominator amongst a much wider array of certified and uncertified masks.

Currently, WHO estimates that 89 million medical masks per month are required to contain the COVID-19 pandemic. There is a severe mask shortage in many countries including the United States, which has the highest number of confirmed COVID-19 cases in the world, and at least a $40 \%$ increase in mask production is required to meet rising global demand (WHO, 2020c). As a result of this shortage, many citizens perceive that they have no other option, and go to the supermarket or the pharmacy or elsewhere with no mask at all. By donning a homemade mask made of any 2-ply or more dense fabric, they would be able shrink their inhalation/exhalation radius immensely and help stem the current outbreaks that are ravaging their country. In addition, cloth masks can be tailor made to fit snugly and close the gaping holes created by poorly fitting surgical masks. Furthermore, a cloth mask can be easily decontaminated as it can be washed with over-the-counter disinfectants, heated in an oven or treated by steam sterilization above $70^{\circ} \mathrm{C}$ for longer than 5 mins, all proven methods to effectively inactivate the SARS-CoV-2 virus (Chin et al., 2020). Surgical masks are designed for single-use and can be decontaminated by any of the above methods, including dry-heating in an electric rice cooker (Lin et al., 2017). However, the potential deformation and degraded effectiveness of the mask's polymer layer after repeated treatments should be further evaluated. With that, the surgical masks are then thrown away and added to the mounting global plastic pollution problem. Thus, the environmental cost of discarded surgical masks is already being outlined and with the persistence of the COVID-19 pandemic and the slow, cautious transition back to normal, this cost will grow unabated unless alternative mask usage is widely promoted. 


\section{CONCLUSIONS}

SARS-CoV-2 transmission is a crucial public health concern and many aspects of this route urgently require further study. For instance, the area of the lungs most vulnerable to SARS-CoV-2 attack is still not fully known. Subsegmental bronchial branches (part of the conducting zone) actually have high levels of ACE2, which SARS-CoV-2 binds to with ease. Because the passageways of the conducting zone are so much larger in diameter than the respiratory zone, large carrier droplets of the virus may be of increased importance. This role of transmission mode (droplet or droplet nuclei) is important to distinguish, but it is not the only factor driving the spread of the virus. The risk of infection calls for a more comprehensive exposure and risk assessment, scrutinizing and integrating all risks including from the source (virus and carrier), in transport (route, aerosol and environmental processes) and at the receptor (host). Considering infected subjects (i.e., source), the expiration range of SARS-CoV-2 aerosols can be effectively attenuated by any mask. Thus, homemade cloth masks should be promoted for use during the pandemic, particularly in countries currently experiencing so-called 'mask shortages'. Cloth masks, just like surgical masks, are expected to reduce our expired air velocity and radius, effectively choking the virus by limiting its spread, while not adding any environmental burden. It is clear that at this moment, we are facing many uncertainties and unknowns about the COVID-19 pandemic, and the outcome could still be catastrophic. Thus, we should draw upon "precautionary principles" and take prudent measures against the virus. In the meantime, the accurate communication and consistent definitions/terminologies among different fields of expertise, as well as their cooperation and collaboration to fill the knowledge gaps are all crucial for controlling and minimizing the virus spread in the near future.

\section{ACKNOWLEDGMENTS}

We are grateful for the motivation, support and collective inputs from a number of distinguished members and the Social Responsibility Committee of Taiwan Association for Aerosol Research.

\section{DISCLAIMER}

The authors declare that they have no conflicts of interest.

\section{REFERENCES}

Asadi, S., Wexler, A.S., Cappa, C.D., Barreda, S., Bouvier, N.M. and Ristenpart, W.D. (2019). Aerosol emission and superemission during human speech increase with voice loudness. Sci. Rep. 9: 2348. https://doi.org/10.1038/s41598-01938808-z

Bourouiba, L. (2020). Turbulent gas clouds and respiratory pathogen emissions: Potential implications for reducing transmission of COVID-19. JAMA https://doi.org/10.1001/jama.2020.4756

Brake, S.J., Barnsley, K., Lu, W., McAlinden, K.D., Eapen, M.S. and Sohal, S.S. (2020). Smoking upregulates angiotensinconverting enzyme-2 receptor: A potential adhesion site for novel Coronavirus SARS-CoV-2 (COVID-19). J. Clin. Med. 9: 841. https://doi.org/10.3390/jcm9030841

Chao, C.Y.H., Wan, M.P., Morawska, L., Johnson, G.R., Ristovski, Z.D., Hargreaves, M., Mengersen, K., Corbett, S., Li, Y., Xie, X. and Katoshevski, D. (2009). Characterization of expiration air jets and droplet size distributions immediately at the mouth opening. J. Aerosol Sci. 40: 122-133. https://doi.org/10.1016/j.jaerosci.2008.10.003

Chen, C.C. and Willeke, K. (1992). Aerosol penetration through surgical masks. Am. J. Infect. Control 20: 177-184. https://doi.org/10.1016/S0196-6553(05)80143-9

Chin, A.W.H., Chu, J.T.S., Perera, M.R.A., Hui, K.P.Y., Yen, H.L., Chan, M.C.W., Peiris, M. and Poon, L.L.M. (2020). Stability of SARS-CoV-2 in different environmental conditions. Lancet Microbe https://doi.org/10.1016/S26665247(20)30003-3

Coutard, B., Valle, C., de Lamballerie, X., Canard, B., Seidah, N.G. and Decroly, E. (2020). The spike glycoprotein of the new coronavirus 2019-nCoV contains a furin-like cleavage site absent in $\mathrm{CoV}$ of the same clade. Antiviral Res. 176: 104742. https://doi.org/10.1016/j.antiviral.2020.104742

Eames, I., Tang, J.W., Li, Y. and Wilson, P. (2009). Airborne transmission of disease in hospitals. J. R. Soc. Interface 6: S697-S702. https://doi.org/10.1098/rsif.2009.0407.focus

Han, Z.Y., Weng, W.G. and Huang, Q.Y. (2013). Characterizations of particle size distribution of the droplets exhaled by sneeze. J. R. Soc. Interface 10: 20130560. https://doi.org/10.1098/rsif.2013.0560

Hoffmann, M., Kleine-Weber, H., Schroeder, S., Kruger, N., Herrler, T., Erichsen, S., Schiergens, T.S., Herrler, G., Wu, N.H., Nitsche, A., Muller, M.A., Drosten, C. and Pohlmann, S. (2020). SARS-CoV-2 cell entry depends on ACE2 and TMPRSS2 and is blocked by a clinically proven protease inhibitor. Cell 181: 271-280. https://doi.org/10.1016/j.cell.2020.02.052

Jung, H., Kim, J., Lee, S., Lee, J., Kim, J., Tsai, P. and Yoon, C. (2014). Comparison of filtration efficiency and pressure drop in anti-yellow sand masks, quarantine masks, medical masks, general masks, and handkerchiefs. Aerosol Air Qual. Res. 14: 991-1002. https://doi.org/10.4209/aaqr.2013.06.0201 
Kim, J.M., Chung, Y.S., Jo, H.J., Lee, N.J., Kim, M.S., Woo, S.H., Park, S., Kim, J.W., Kim, H.M. and Han, M.G. (2020). Identification of coronavirus isolated from a patient in Korea with COVID-19. Osong Public Health Res. Perspect. 11: 3-7. https://doi.org/10.24171/j.phrp.2020.11.1.02

Lee, B.U., Yermakov, M. and Grinshpun, S.A. (2005). Filtering efficiency of N95- and R95-type facepiece respirators, dustmist facepiece respirators, and surgical masks operating in unipolarly ionized indoor air environments. Aerosol Air Qual. Res. 5: 25-38. https://doi.org/10.4209/aaqr.2005.06.0003

Li, F., Li, W., Farzan, M. and Harrison, S.C. (2005). Structure of SARS coronavirus spike receptor-binding domain complexed with receptor. Science 309: 1864-1868. https://doi.org/10.1126/science.1116480

Li, Y., Leung, G.M., Tang, J.W., Yang, X., Chao, C.Y., Lin, J.Z., Lu, J.W., Nielsen, P.V., Niu, J., Qian, H., Sleigh, A.C., Su, H.J., Sundell, J., Wong, T.W. and Yuen, P.L. (2007). Role of ventilation in airborne transmission of infectious agents in the built environment - A multidisciplinary systematic review. Indoor Air 17: 2-18. https://doi.org/10.1111/j.16000668.2006.00445.x

Lin, T.H., Chen, C.C., Huang, S.H., Kuo, C.W., Lai, C.Y., and Lin, W.Y. (2017). Filter quality of electret masks in filtering 14.6-594 nm aerosol particles: Effects of five decontamination methods. PLoS One 12: e0186217. https://doi.org/10.1371/ journal.pone.0186217

Lin, T.H., Tseng, C.C., Huang, Y.L., Lin, H.C., Lai, C.Y. and Lee, S.A. (2020). Effectiveness of N95 facepiece respirators in filtering aerosol following storage and sterilization. Aerosol Air Qual. Res. 20: 833-843. https://doi.org/10.4209/aaqr. 2019.12.0620

Liu, L., Wei, J., Li, Y. and Ooi, A. (2017). Evaporation and dispersion of respiratory droplets from coughing. Indoor Air 27: 179-190. https://doi.org/10.1111/ina.12297

Lukassen, S., Chua, R.L., Trefzer, T., Kahn, N.C., Schneider, M.A., Muley, T., Winter, H., Meister, M., Veith, C., Boots, A.W., Hennig, B.P., Kreuter, M., Conrad, C. and Eils, R. (2020). SARS-CoV-2 receptor ACE2 and TMPRSS2 are primarily expressed in bronchial transient secretory cells. EMBO J. 14: e105114. https://doi.org/10.15252/embj.20105114

Morawska, L., Johnson, G.R., Ristovski, Z.D., Hargreaves, M., Mengersen, K., Corbett, S., Chao, C.Y.H., Li, Y. and Katoshevski, D. (2009). Size distribution and sites of origin of droplets expelled from the human respiratory tract during expiratory activities. J. Aerosol Sci. 40: 256-269. https://doi.org/10.1016/j.jaerosci.2008.11.002

Nicas, M., Nazaroff, W.W. and Hubbard, A. (2005). Toward understanding the risk of secondary airborne infection: Emission of respirable pathogens. J. Occup. Environ. Hyg. 2: 143-154. https://doi.org/10.1080/15459620590918466

Oberg, T. and Brosseau, L.M. (2008). Surgical mask filter and fit performance. Am. J. Infect. Control 36: $276-282$. https://doi.org/10.1016/j.ajic.2007.07.008

Ollier, K.J. (2018). Inhalation exposure and respiratory protection of home healthcare workers administering aerosolized medications (simulation study). Aerosol Air Qual. Res. 19: 937-944. https://doi.org/10.4209/aaqr.2018.10.0366

Satija, N. and Lal, S.K. (2007). The molecular biology of SARS coronavirus. Ann. N.Y. Acad. Sci. 1102: $26-38$. https://doi.org/10.1196/annals.1408.002

U.S. Centers for Disease Control and Prevention (CDC) (2012). Principles of Epidemiology, 3rd ed., U.S. Department of Health and Human Services, Atlanta.

van Doremalen, N., Bushmaker, T., Morris, D.H., Holbrook, M.G., Gamble, A., Williamson, B.N., Tamin, A., Harcourt, J.L., Thornburg, N.J., Gerber, S.I., Lloyd-Smith, J.O., de Wit, E. and Munster, V.J. (2020). Aerosol and surface stability of SARS-CoV-2 as compared with SARS-CoV-1. N. Engl. J. Med. 382: 1564-1567. https://doi.org/10.1056/NEJMc2004973

Vankadari, N. and Wilce, J.A. (2020). Emerging WuHan (COVID-19) coronavirus: Glycan shield and structure prediction of spike glycoprotein and its interaction with human CD26. Emerging Microbes Infect. 9: 601-604. https://doi.org/10.1080/ 22221751.2020 .1739565

Vejerano, E.P. and Marr, L.C. (2018). Physico-chemical characteristics of evaporating respiratory fluid droplets. J. R. Soc. Interface 15: 20170939. https://doi.org/10.1098/rsif.2017.0939

Wells, W.F. (1934). On air-borne infection study: II - Droplets and droplet nuclei. Am. J. Hyg. 20: 619-627. https://doi.org/ 10.1093/oxfordjournals.aje.a118097

World Health Organization (WHO) (2020a). Advice on the use of masks in the context of COVID-19: Interim guidance. April 6, 2020. World Health Organization. https://apps.who.int/iris/handle/10665/331693. License: CC BY-NC-SA 3.0 IGO

World Health Organization (WHO) (2020b). Coronavirus disease 2019 (COVID-19): Situation Report - 66, March 26, 2020, World Health Organization, https://www.who.int/docs/default-source/coronaviruse/situation-reports/20200326-sitrep66-covid-19.pdf?sfvrsn=9e5b8b48_2

World Health Organization (WHO) (2020c). Shortage of personal protective equipment endangering health workers worldwide. In Newsroom, March 3, 2020, World Health Organization. https://www.who.int/news-room/detail/03-032020-shortage-of-personal-protective-equipment-endangering-health-workers-worldwide

Wrapp, D., Wang, N., Corbett, K.S., Goldsmith, J.A., Hsieh, C.L., Abiona, O., Graham, B.S. and McLellan, J.S. (2020). Cryo-EM structure of the 2019-nCoV spike in the prefusion conformation. Science 367: 1260-1263. https://doi.org/10.1126/ science.abb2507

Xie, X., Li, Y., Chwang, A.T., Ho, P.L. and Seto, W.H. (2007). How far droplets can move in indoor environments revisiting the Wells evaporation-falling curve. Indoor Air 17: 211-225. https://doi.org/10.1111/j.1600-0668.2007.00469.x. 
Yang, S., Lee, G.W., Chen, C.M., Wu, C.C. and Yu, K.P. (2007). The size and concentration of droplets generated by coughing in human subjects. J. Aerosol Med. 20: 484-494. https://doi.org/10.1089/jam.2007.0610

Yu, I.T., Li, Y., Wong, T.W., Tam, W., Chan, A.T., Lee, J.H., Leung, D.Y. and Ho, T. (2004). Evidence of airborne transmission of the severe acute respiratory syndrome virus. N. Engl. J. Med. 350: 1731-1739. https://doi.org/10.1056/NE JMoa032867

Zhou, Y. and Cheng, Y.S. (2016). Evaluation of N95 filtering facepiece respirators challenged with engineered nanoparticles. Aerosol Air Qual. Res. 16: 212-220. https://doi.org/10.4209/aaqr.2015.06.0399

Zou, L., Ruan, F., Huang, M., Liang, L., Huang, H., Hong, Z., Yu, J., Kang, M., Song, Y., Xia, J., Guo, Q., Song, T., He, J., Yen, H.L., Peiris, M. and Wu, J. (2020). SARS-CoV-2 viral load in upper respiratory specimens of infected patients. $N$. Engl. J. Med. 382: 1177-1179. https://doi.org/10.1056/NEJMc2001737

Received for review, April 17, 2020

Revised, April 25, 2020

Accepted, April 25, 2020 